\title{
Impact of CCSDS-IDC and JPEG 2000 Compression on Image Quality and Classification
}

\author{
Alaitz Zabala, ${ }^{1,2}$ Raffaele Vitulli, ${ }^{2}$ and Xavier Pons ${ }^{1}$ \\ ${ }^{1}$ Department of Geography, Universitat Autònoma de Barcelona, 08193 Cerdanyola del Vallès, Spain \\ ${ }^{2}$ European Space Agency, ESTEC, 2201 AZ Noordwijk ZH, The Netherlands \\ Correspondence should be addressed to Alaitz Zabala, alaitz.zabala@uab.cat
}

Received 17 June 2011; Revised 29 September 2011; Accepted 2 November 2011

Academic Editor: Bruno Aiazzi

Copyright ( 2012 Alaitz Zabala et al. This is an open access article distributed under the Creative Commons Attribution License, which permits unrestricted use, distribution, and reproduction in any medium, provided the original work is properly cited.

\begin{abstract}
This study measures the impact of both on-board and user-side lossy image compression (CCSDS-IDC and JPEG 2000) on image quality and classification. The Sentinel-2 Image Performance Simulator was modified to include these compression algorithms in order to produce Sentinel-2 simulated images with on-board lossy compression. A multitemporal set of Landsat images was used for the user-side compression scenario in order to study a crop area. The performance of several compressors was evaluated by computing the Signal-to-Noise Ratio (SNR) of the compressed images. The overall accuracy of land-cover classifications of these images was also evaluated. The results show that on-board CCSDS performs better than JPEG 2000 in terms of compression fidelity, especially at lower compression ratios (from CR 2:1 up to CR 4:1, i.e., 8 to 4 bpppb). The effect of compression on land cover classification follows the same trends, but compression fidelity may not be enough to assess the impact of compression on enduser applications. If compression is applied by end-users, the results show that 3D-JPEG 2000 obtains higher compression fidelity than CCSDS and JPEG 2000 with other parameterizations. This is due to the high dynamic range of the images (representing reflectances $* 10000$ ), which JPEG 2000 is able to exploit better.
\end{abstract}

\section{Introduction}

Remote Sensing (RS) images are used for many applications, including land cover mapping and analysis, disaster management, climate modelling, and agricultural and forest management. The recent trend in RS satellites is characterized by an increase in resolution (spatial, spectral, and temporal) of the new satellite sensors. Unfortunately, this increase has to be kept inside the downlink capacity of the mission, or onboard compression needs to be applied. Lossless compression is applied on board, but lossy compression is also considered as a possible solution when lossless compression is not enough [1]. From the user perspective, this improvement in the sensors is greatly appreciated as it provides a large amount of data; however, at the same time it leads to management problems, especially when large time series are used.

The new Spatial Data Infrastructure (SDI) paradigm developed over recent years promotes the establishment of web data services, usually in terms of Open Geospatial Consortium standards like the Web Map Service (WMS), the Web Coverage Service (WCS) [2], or the recent Web Map Tile Service (WMTS) [3]. However, it is necessary to use compression and interactive transmission strategies for these web services in order to transfer images (which may be very large in the case of WCS), repetitively, to environments with restricted bandwidth. It is also necessary to standardize data compression and transmission formats in SDI environments in order to make interoperability possible. Thus, the use of standard compression formats is unavoidable even if it implies slightly worse compression than that obtained with new but nonstandard algorithms recently published.

Previous research on image compression (usually without taking into account the effects on the user's application) concluded that wavelet-based algorithms, such as JPEG 2000 (ISO standard since 2000, revised in 2004 [4]), obtain better results than those based on discrete cosine transform (DCT), such as JPEG. Thus, JPEG 2000 seems the option that should be studied further in terms of the effect of compression on the final user application, such as crop classification. The Consultative Committee for Space Data Systems (CCSDSs) has been developing space data handling standards for onboard compression since 1982. The Image Data Compression (IDC) recommendation (CCSDS 122.0-B-1 [5]) is a lossless and lossy compression standard designed specifically for 
use on board a space platform. This standard has sought a balance between the complexity of the algorithm and its performance, and thus it can be implemented by hardware and software more easily. The CCSDS-IDC encoding process is divided in two parts. First a 2D-discrete wavelet transform (with three DWT levels) is performed in order to decorrelate the original data. Then the transformed data are rearranged in $8 \times 8$ blocks that are grouped in segments. Finally each segment is independently encoded by the bit-plane encoder.

The main aim of this work is to compare the impact of the CCSDS and JPEG 2000 compression standards, paying particular attention to both payload data processing and user-side applications. This double approach can be applied in order to determine both the moment in the processing chain when compression should be applied (on-board or at the end of the processing chain) and the analysis to be performed (noise evaluation or final application assessment). The effects of both on board and user-side compressions were studied in terms of compression fidelity and an applied approach, that is, image classification.

\section{Methods and Scenes Used}

Two different scenarios have been studied in this paper: The first scenario evaluates the effect of on-board compression and the second scenario the effect of user compression. In both cases the compression performance was evaluated using noise evaluation and a final application assessment. In the following subsections we explain the methods and scenes used for the two scenarios (on-board compressionSection 2.1-and user-side compression-Section 2.2) and the methods for evaluating the impact of compression in the two scenarios (Section 2.3).

In this paper, the compression ratio (CR) is computed as the ratio between the size of the original file and the size of the compressed file and is expressed as, for example, 10:1 for a file that is compressed to a tenth of the original file size. This measure is used because it is more intuitive for a more general remote sensing user community that is not used to other compression measures such as bit rates (BRs) in bits per pixel per band (bpppb). Moreover, in the SNR graphics, the compression measure is also expressed using bit rates in bpppb to allow comparison with compression literature. CR and BR measures are inversely proportional. It is necessary to mention that all images used on the paper have an initial bitdepth of 16 bpppb, thus a CR $2: 1$ is equivalent to a bit rate of 8 bpppb, or a CR 5 is equivalent to a bit are of 3.2 bpppb.

\subsection{On-Board Compression}

2.1.1. Overview of the Sentinel-2 Mission and the Sentinel2 Image Performance Simulator. To assess the effect of onboard image compression, we used the instrument simulators developed to assess mission requirements. The Sentinel2 optical mission $[1,6]$ is part of the Global Monitoring for Environment and Security (GMES) system, which is a joint initiative of the European Commission (EC) and the European Space Agency (ESA) designed to develop the European capacity for providing and using operational monitoring information for environment and security applications. Sentinel-2 polar-orbiting satellites provide systematic global acquisitions of high-resolution multispectral imagery with a high revisit frequency. The optical payload, the Multispectral Imager (MSI), will acquire scenes at three simultaneous resolutions $(10 \mathrm{~m}, 20 \mathrm{~m}$, and $60 \mathrm{~m})$ in the VNIR and SWIR ranges over a wide swath of around $290 \mathrm{~km}$.

The Sentinel-2 Image Performance Simulator [7] was developed by EADS Astrium. It produces simulated Sentinel2 images starting from hyperspectral images with an adequate spectral configuration (covering all bands of the MSI instrument) whose pixels represent the bottom of the atmosphere's reflectance. The simulator is able to simulate Sentinel-2 images using on-board compression at several compression ratios. The compression and decompression during the image simulation is performed by calling external executables. As our main concern is to compute the performance of CCSDS and JPEG 2000 when they are used on board, the simulator has been modified to allow the user to select from among three compression choices: CCSDS, JPEG 2000, and the compressor that was originally included (nonstandard, developed by CNES).

The simulator was developed to produce images which can be delivered from the ground segment at several postprocessing levels, from observed rough data products to estimated geophysical end products. In this study we generate simulated images at level $1 \mathrm{~b}$ by applying geometric and radiometric calibration corresponding to ground segment processing to obtain images that are ready to be georegistered and georeferenced. Thus, the simulation includes on-board acquisition and compression as well as ground segment processing.

2.1.2. Areas and Scenes: CEFLES-2 Campaign. CEFLES-2 (CarboEurope, FLEX and Sentinel-2) was conceived as a collective multiobjective campaign to exploit the synergies between the three experiments that were collocated in the Les Landes region of France from April to September 2007 [8]. It focused on various landscape types, including urban, agricultural, water, and forested areas. The campaign was mainly based on optical airborne and satellite acquisitions and the dataset obtained was completed with several onground observations and measurements.

The airborne hyperspectral acquisitions were considered as the main input for the simulation phase. The Airborne Hyperspectral Scanner (AHS) is an 80-band airborne imaging radiometer developed and built by SensyTech Inc. (currently Argon ST, and formerly Daedalus Ent. Inc.) and is operated by the Spanish Institute for Aerospace Technology (INTA) in different remote sensing projects. It has 63 bands in the reflective part of the electromagnetic spectrum, 7 bands in the 3 to $5 \mu \mathrm{m}$ range, and 10 bands in the 8 to $13 \mu \mathrm{m}$ region.

The study uses four AHS images (see Figure 1) from the third mission of the CEFLES-2 campaign (September 2007), covering areas with different landscapes (urban, crops, forest, and mixed areas). Les Landes is characterized by an agricultural environment with different types of crops and 


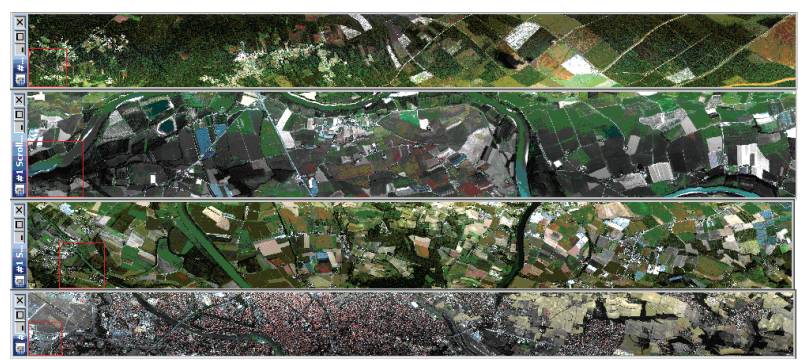

Figure 1: True color composition of AHS images acquired in the CEFLES-2 campaign (mission 3, September 2009). From top to bottom: "Les Landes-P01BS", "Les Landes-P03BS", "ToulouseForest-F01BS," and "Toulouse-Urban-T01BS.

fields located around very small urban centers. Two images of this area were selected. The first one covers a tree crop area (P01BS) and the second one covers a nontree crop area (P03BS) for which ground truth is available. Images over Toulouse were sensed over very urbanized areas (T01BS), including city outskirts mainly characterized by asphalted areas and industrial zones, and also a forested area (F01BS). For the selected urban area the ground truth can be digitized by means of photo interpretation.

2.1.3. Compression. The ESA CCSDS compression implementation [9] was used to compress the images using the CCSDS standard. This implementation does not have a command line application for decompressing the images, and therefore the University of Nebraska-Lincoln implementation [10] was used for decompression. The JPEG 2000 compression implementation of the Autonomous University of Barcelona (GICI group) [11], called BOÍ, was used to compress and decompress images using the JPEG 2000 standard. Moreover, the CNES internal compressor (a nonstandard compression algorithm, included in the original Sentinel-2 image performance simulator) was used in order to compare the results obtained with those obtained with previous standardized options. Details about how CNES compressor works cannot be disclosed because it is covered by a patent.

Two strategies were used to distribute the bit rate (defined for each band) among bands. The first strategy uses the same target compression ratio (CR, i.e., the same bit rate) for all the bands. The second strategy applies more compression to the $10 \mathrm{~m}$ bands and less compression to the $20 \mathrm{~m}$ and $60 \mathrm{~m}$ bands in order to obtain the same overall target compression ratio. The proportion of the compression ratio applied to each band follows the proportions usually used in Earth Observation scenarios of CR 4.0 for the $10 \mathrm{~m}$ bands and CR 2.2 for the other bands. Taking into account these values and the spatial and spectral configuration of Sentinel-2, the proportion was set to 0.6776 and 1.2320 of the desired CR to be applied to the $20-60 \mathrm{~m}$ and $10 \mathrm{~m}$ bands, respectively. The compression ratio applied to each band (and the bit rates used) at several compression ratios is shown in Table 1.

The first CRs are those studied for the Sentinel-2 mission. Although CR 10 and 20 are out of the scope of Sentinel-2, they were also studied in order to determine their impact on classification. For these higher compression ratios only the equally distributed bit rates were selected because they are out of the scope of on-board Earth Observation missions, and therefore the previous rule does not need to be applied.

2.1.4. Classification. Some processing needs to be carried out after image simulation (with or without compression) and before image classification. The simulated bands that will be used in the classification $(10 \mathrm{~m}$ and $20 \mathrm{~m}$ bands for "Les Landes-P03BS" and "Toulouse-Urban-T01BS" scenes) need to be registered to the desired projection system, stacked in one file and finally used for the classification. All these processes are included in an IDL routine and can be applied easily to each of the desired scenarios (different scenes, compression ratios, and compression algorithms). Registration was performed using the MiraMon software [12] by defining ground control points in each set of images $(10 \mathrm{~m}$ and $20 \mathrm{~m})$ and by batch processing. The ENVI Layer Stacking basic tool was used to create a single BSQ file with all bands with a pixel size of $20 \mathrm{~m}$, with a cubic convolution resampling method. The resulting file was used to feed the classifier algorithm (Maximum Likelihood) using ENVI software [13]. It is important to bear in mind that when compressed images are classified, the classifier is trained with the actual lossy compressed data that will be classified, not with the original uncompressed data. Moreover, we wanted to point out we computed the histograms of original (noncompressed) images and the histograms of compressed images inside the training areas to prove that they generally fit to a Gaussian model and, thus, it is feasible to use a classifier such as Maximum Likelihood.

Training and test areas are needed to execute the supervised classification and to evaluate it. For the "Les Landes-P03BS" scene, the CEFLES-2 campaign included ground truth information regarding crops in the area. Using this information, the entire set of ground truth data was manually digitized in the original AHS image. 165 polygons were digitized as ground truth, covering a total area of 218 ha (more than $10 \%$ of the area covered by the image). The target legend for this area includes corn, trees, plastic foils, wheat, wild grass, fruit trees, beans, wheat mixture, water, and bare soil. However, no ground truth information was originally available for the "Toulouse-Urban-T01BS" scene. Thus, a general classification was carried out by manually digitizing the categories to be classified in the original AHS image. 165 polygons were digitized as the ground truth, covering a total area of 135 ha (about $4 \%$ of the area covered by the image). The target legend for the Toulouse scene includes water, trees, gardens, soil, agricultural fields, asphalt, and buildings. The ground-truth information (of each scene) was randomly split into two sets, one for training the classifier $(70 \%$ of the ground-truth) and the other for carrying out an independent evaluation test (remaining 30\%). Each spatially disjoint field was assigned to a training or test set in order to avoid a chessboard distribution of the training and test pixels, which would generate strongly correlated and positively biased estimations of the probability of correct classification.

2.2. User-Side Compression. To assess the impact of the CCSDS and JPEG 2000 compression standards, it is also 
TABle 1: Compression ratio (CR, expressed as $x: 1$, e.g., $5: 1)$ and distribution among bands: two approaches were used. Moreover bit rates in bpppb are also indicated (compressed images inside the simulator have a bit-depth of 16 bpppb).

\begin{tabular}{lccccccccc}
\hline Target CR $(x: 1)$ & 2 & 2.39 & 3 & 3.25 & 4 & 4.79 & 5 & 10 & 20 \\
bit-rate (bpppb) & $(8)$ & $(6.69)$ & $(5.33)$ & $(4.92)$ & $(4)$ & $(3.34)$ & $(3.2)$ & $(1.6)$ & $(0.8)$ \\
\hline 10 m unequally & 2.46404 & 2.94453 & 3.69607 & 4 & 4.92809 & 5.90138 & 6.16011 & - \\
$20-60$ m unequally & 1.35522 & 1.61949 & 2.03284 & 2.2 & 2.71045 & 3.24576 & 3.38806 & - \\
10 m equally & 2 & 2.39 & 3 & 3.25 & 4 & 4.79 & 5 & 10 & 20 \\
$20-60$ m equally & 2 & 2.39 & 3 & 3.25 & 4 & 4.79 & 5 & 10 & 20 \\
\hline
\end{tabular}

necessary to consider the scenario in which compression is applied at the very end of the image processing chain, that is, just before the final end-user application. In this scenario, the aim is to assess the influence of lossy image compression on digital classification applied to crop areas in a real management environment, that is, a multitemporal set of images that covers a wide area. This study focuses on crop areas because of their economic and human interest and because of the challenge they represent. However, to obtain the best results it is advisable to mask agricultural fields due to their heterogeneity [14].

2.2.1. Areas and Scenes. Two medium-sized zones were chosen, located in two different agricultural regions: Segrià and Pla d'Urgell (see Figure 2). The Segrià zone has more fruit trees and is slightly less fragmented (see Figure 3). The Pla d'Urgell zone has more maize and is somewhat more fragmented, and irrigated herbaceous crops and dry permanent crops predominate in this area.

Both zones are in Catalonia (NE Spain). They were analyzed using Landsat-5 TM images, which are especially suitable for producing land cover maps due to their spatial, temporal and spectral resolution [15]. Images were geometrically corrected, resampled (using the nearest neighbor to preserve the original radiometry) [16], and processed to convert digital numbers to reflectance values [17]. Landsat TM images store each value band in bytes; however, radiometric correction produces short integer images (reflectances * 10000, 16 bpppb).

The dimensions of the fragment of the TM scenes covering the study zones were $1517 \times 1311$ pixels $(53585$ ha of crops) for Segrià and $1307 \times 1059$ (50099 ha) for Pla d'Urgell. Both were analyzed using images from 16-05-2004, 17-06-2004, 19-07-2004, 23-10-2004, and 08-11-2004. These images were selected from those available for 2004 (without clouds) to include the different spectral responses over the year due to the phenological state of the crop.

2.2.2. Compression. CCSDS and JPEG 2000 were both considered in order to measure the effect of compression on the classification results. Unlike in the on-board compression scenario (Section 2.1.3), in this scenario not only monoband compression was used but also multiband and three-dimensional (3D) compression were analyzed with the appropriate software. The compression algorithms used were the same as those used within the simulator as well as
Kakadu [18] to perform 3D JPEG 2000 compression (i.e., a decorrelation method was applied not only in the spatial domain but also in the spectral domain).

The CCSDS compression standard in image frame mode allows the user to compress each band independently. As the images we are dealing with have six bands (the TM thermal band was not used), we decided to compress each band separately to the desired compression ratios (hereafter CCSDS). JPEG 2000 compression allows monoand multiband compression. Thus, several strategies were used for JPEG 2000 compression. To obtain comparable results for the two compression standards, a first JPEG 2000 approach compressed each band independently (hereafter $\mathrm{J} 2 \mathrm{~K})$. Then, two more approaches were used: a multiband compression that compresses all the bands of each image (each date) in a single JPEG 2000 file (hereafter J2Km, without compression among bands), and the last scenario using a 3D JPEG 2000 compression (hereafter J2Km_3D, using spectral-3D-compression) to make full use of the JPEG 2000 capabilities. The compression ratios studied were 2, $2.39,3,4,4.79,5,6,7,8,9,10,15,20,50$, and 100 (corresponding to bit rates of: 8, 6.69, 5.33, 4.92, 4, 3.34, 3.2, 1.6 , and 0.8 bpppb, respectively).

The original images have areas without data (NODATA) due to the radiometric corrections carried out on them and the presence of a small number of clouds. The compression/decompression programs that were used are currently unable to recognize these NODATA values. Using these values as useful values when an image is compressed will generate serious errors in the compressed image, especially if the NODATA value is an extreme value of the available rank, which is very usual. According to the existing literature, the JPEG 2000 compliant approach that obtains the highest compression fidelity is the one that substitutes NODATA values with arithmetical means of the whole image before it is compressed [19]. Finally, it is necessary to create a mask with the NODATA areas in the original images (different for each date and band) that can be reapplied to the images after decompression.

2.2.3. Classification. In order to classify only the crop areas, a mask obtained from the Land Cover Map of Catalonia was applied (as determined by [14]). Two classification methods were fed with the five available images, that is, 30 bands. The first one (hereafter IsoMM) is a hybrid classifier that combines an unsupervised classifier and a nonparametric 


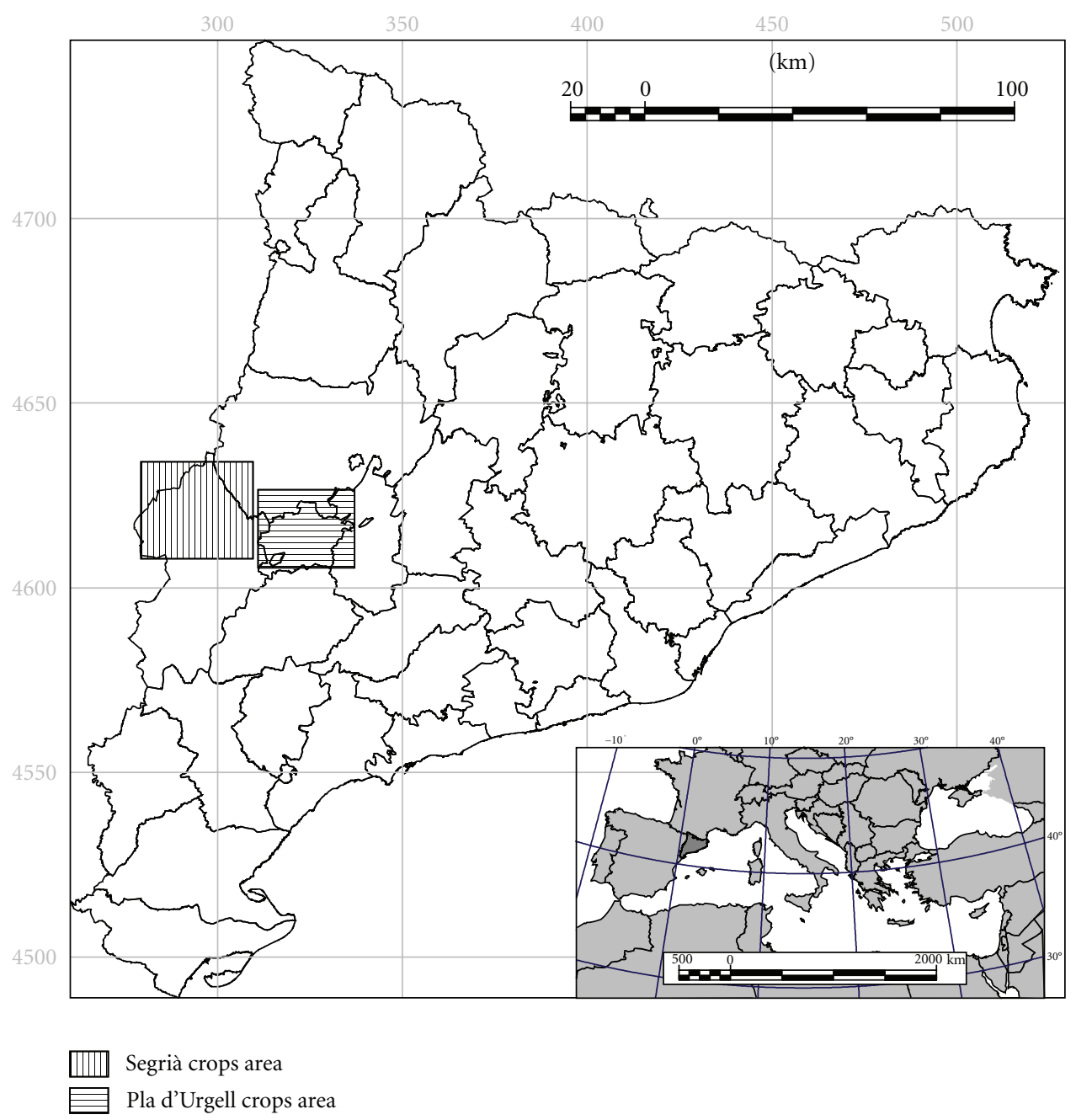

Figure 2: Zones used in the study located in Catalonia (northeast Spain). Two zones were selected: Segrià and Pla d'Urgell.

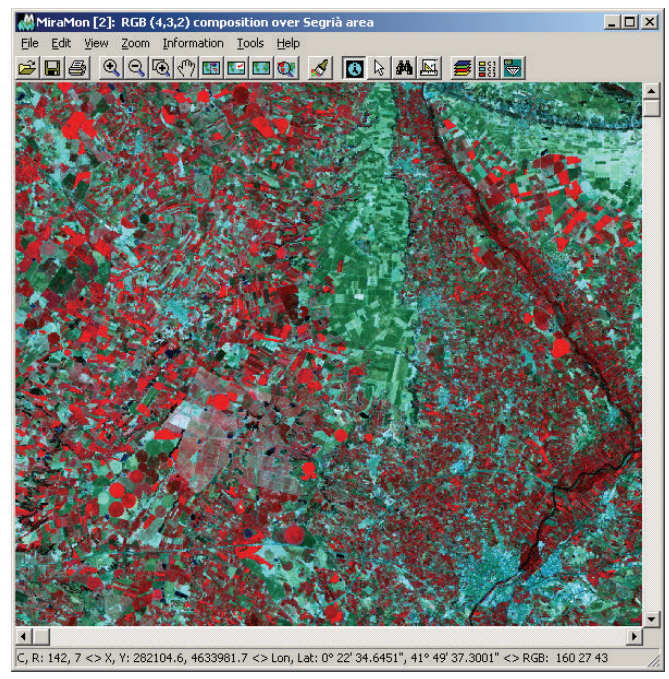

(a)

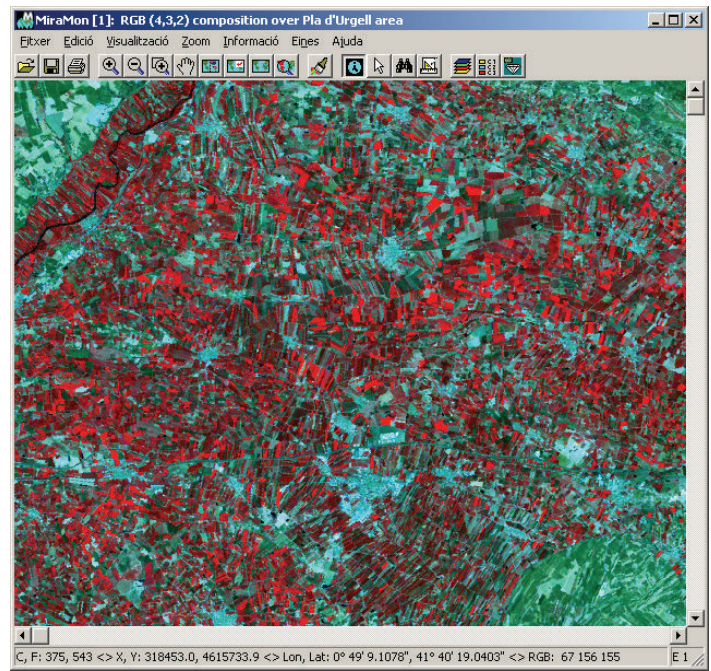

(b)

FIGURE 3: False color RGB composition (NIR, red and green bands of the 17th of June scene) of both studied areas: Segrià (a) and Pla d'Urgell (b). 
supervised classifier [20, 21] implemented in MiraMon software [12]. Moreover, classic Maximum Likelihood general classifier (ENVI implementation [13]) was also used (hereafter MaxLike). According to the on-board compression scenario, the classifiers were trained with the same lossy compressed data that will be classified. Moreover, normality of data has been tested in order to guarantee the feasibility of using a classifier such as Maximum Likelihood.

2.3. Evaluation of Compression Effects. The performance of several compressors and compression ratios was evaluated using compression noise evaluation and a final application assessment. First, the SNR (Signal-to-Noise Ratio) of the compressed images (in relation to the simulated or original image without compression) was computed and then the overall accuracy of the land-cover classifications of the images was used.

2.3.1. Compression Noise Evaluation Method. To evaluate compression noise, original and simulated images (without compression) were compared to those obtained by applying compression algorithms. The Gcomp application (developed by the GICI group of the Autonomous University of Barcelona) was used to compute the SNR.

2.3.2. Classification Evaluation Method. The classifications obtained (from images with or without compression) were evaluated using the confusion matrix [22] based on groundtruth test areas that are independent from the training areas used in the supervised classification stage. Using test areas (covering a small percentage of the classified area) is a common approach for evaluating classifications (i.e., in [23]) because it employs independent information not used in the classification, and is not very time consuming. Moreover, when compression effects are evaluated, it is also usual to compare classifications of compressed images with the classifications of noncompressed images (i.e., in [24]). This evaluation method has a high comparison area, which allows more robust results to be obtained. Thus, the confusion matrix was also obtained using the classification of noncompressed images as ground truth (instead of test areas). These two complementary evaluation approaches enhance the comparability of this study with those published earlier.

\section{Results}

\subsection{Compression Noise Evaluation Results}

3.1.1. On-Board Compression. Compression noise was assessed by computing the SNR between L1B bands simulated with lossy compression with L1B bands simulated with lossless compression (both using instrument noise). Several compression ratios and two approaches for distributing compression among bands were studied.

Only a few values were available for the CNES compressor, and therefore the results are presented in two different sets. First, Figure 4 and Table 2 show the mean SNR obtained by all the bands of each simulated image using CCSDS and JPEG 2000 compressions at several compression ratios. In the figure (showing compression ratios up to $\mathrm{CR}$ 5, i.e., 3.2 bpppb), line color indicates the compression algorithm (red or orange for CCSDS and blue for JPEG 2000), while line style depicts whether the bit rate was distributed unequally or equally among bands (continuous and dashed, respectively). The table shows the results for the higher compression ratios studied (CR 5 to 20, i.e., bit rates from 3.2 to $0.8 \mathrm{bpppb}$ ). CR 5 appears in both the figure and the table so they can be easily related.

The results of the CCSDS and JPEG 2000 compressors clearly indicate that CCSDS performs better than JPEG 2000 at lower compression ratios (up to CR 5 : 1, i.e., 3.2 bpppb, see Figure 4). JPEG 2000 performs better than CCSDS if higher CRs are used (10 or 20, i.e., less than $1.6 \mathrm{bpppb}$, see Table 2). This trend is found both for an unequal and equal bit rate distribution among bands. The two approaches were compared and it was found that unequally distributed scenarios clearly obtain a higher all-band mean SNR. It is difficult to compare the CNES compressor with CCSDS and JPEG 2000 because only a few mean values of all bands are available for the CNES scenarios. Thus, the presented comparison is based on $10 \mathrm{~m}$ bands, which are the subset of bands with the most available CNES results. The SNR of the $10 \mathrm{~m}$ bands for all compression algorithms is presented in Figure 5. As expected, in this case the equal distribution of bit rate obtains better results because it implies that less compression is applied to these bands (i.e., for CR 2, i.e., 8 bpppb, when unequally distributed BR is used, the actual CR applied to $10 \mathrm{~m}$ bands is 2.464, i.e., 6.49 bpppb, see Table 1). In most cases CNES compression obtains a lower compression fidelity than either of the other compression standards. Moreover, as this is not a standardized algorithm, using it on board should be avoided.

Interestingly, in some cases, and despite the compression standard and ratio applied, the SNR results are the same up to medium compression (up to 5.9 depending on the band and scene). The number of scenarios with the same SNR can be explained taking into account spatial resolution and the dynamic range of each band (the larger the pixel size or the lower the dynamic range, the more compression ratios that will have the same PSNR). Interestingly, this order is also related to the difference between JPEG 2000 and CCCSD SNR values. Thus, for the same spatial resolution, the higher the dynamic range, the smaller the difference between the two compression standards.

3.1.2. User-Side Compression. Figure 6 shows the mean SNR for all the bands computed for both of the crop scenes used at several compression ratios and using the four studied compression options. In this case, CCSDS gives the worst results. However, as expected the more JPEG 2000 features used, the better the results obtained. Between areas, the Pla d'Urgell area obtains a smaller SNR, which makes sense if we take area fragmentation into account because the Pla d'Urgell is the most fragmented area. Comparing these results with those obtained in the previous section (onboard compression), it seems that they are not consistent. But if we take a careful look at them, we find that the higher the dynamic range, the smaller the difference between 


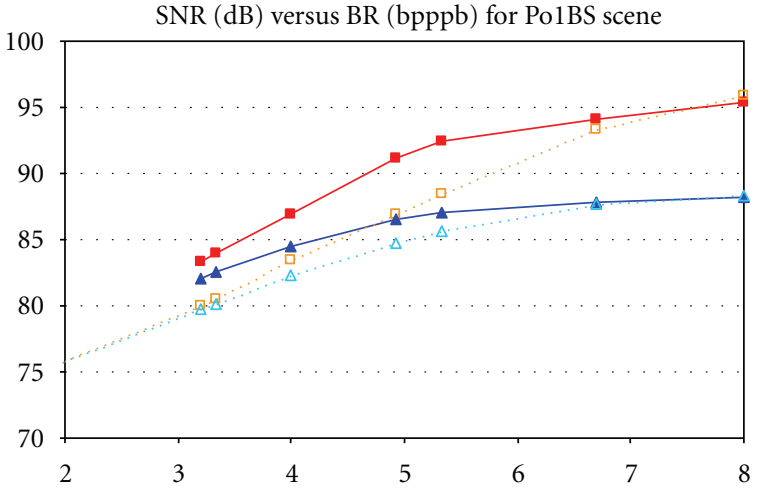

(a)

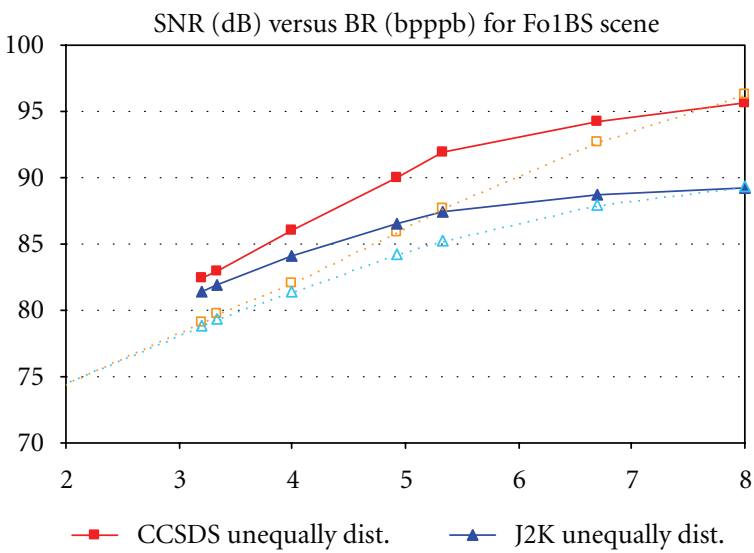

(c)

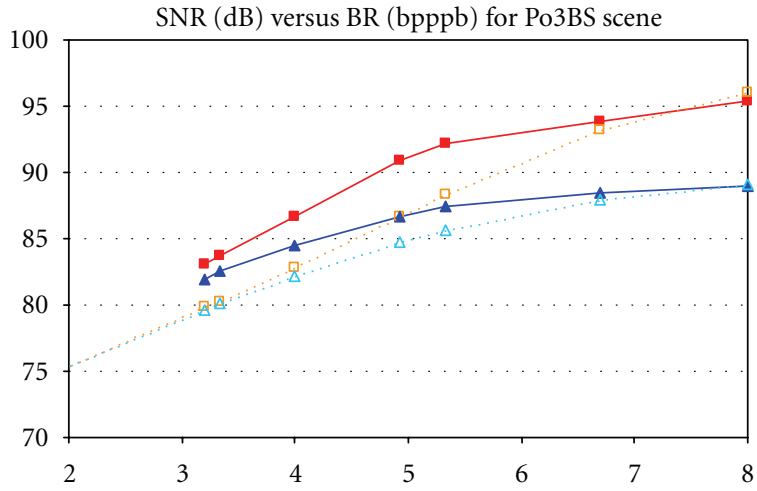

(b)

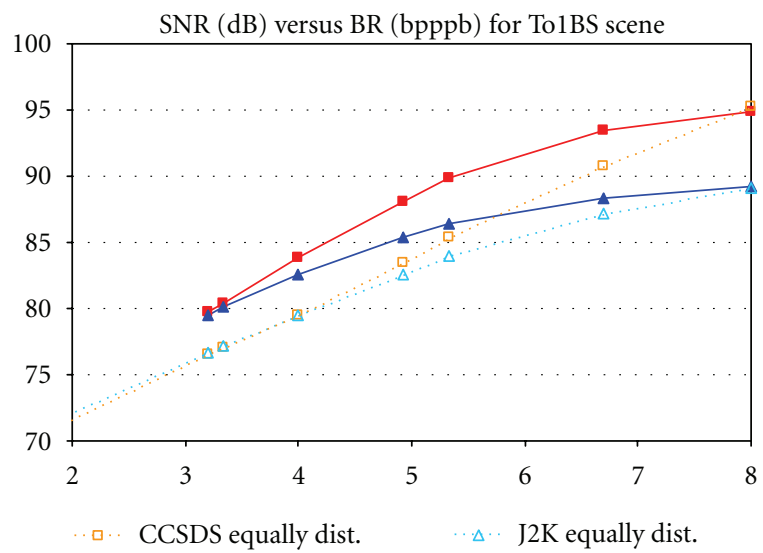

(d)

FIgURE 4: SNR (in dB) in relation to the bit rate (in bpppb) of CCSDS and JPEG 2000 for all scenes at high bit rates. Two strategies were used to split BR among bands (at each compression ratio): unequally or equally distributed. (Please note that the $y$-axis is not the same as in Figures 5 and 6.)

TABLE 2: SNR (in dB) in relation to the bit rate (in bpppb) of CCSDS and JPEG 2000 for all scenes at low bit rates. Only the equally distributed $\mathrm{BR}$ among bands was studied at these compression ratios.

\begin{tabular}{lcccccccc}
\hline \multirow{2}{*}{ Bit rate } & \multicolumn{2}{c}{ P01BS } & \multicolumn{2}{c}{ P03BS } & \multicolumn{2}{c}{ F01BS } & \multicolumn{2}{c}{ T01BS } \\
& CCSDS & JPEG 2000 & CCSDS & JPEG 2000 & CCSDS & JPEG 2000 & CCSDS & JPEG 2000 \\
\hline 3.2 & 80.0177 & 79.7148 & 79.8104 & 79.5801 & 79.1473 & 78.8766 & 76.4946 \\
1.6 & 74.4612 & 74.4679 & 73.8812 & 74.0286 & 72.9307 & 73.0981 & 70.0467 & 70.5904 \\
0.8 & 71.2322 & 71.2668 & 69.8731 & 70.0815 & 69.0672 & 69.0926 & 66.4980 & 66.8458 \\
\hline
\end{tabular}

CCSDS and JPEG 2000. Sentinel-2 L0 bands have a very small dynamic range that may be exploited better by CCSDS than by JPEG 2000, but as the dynamic range increases (and it is quite high in the Landsat images representing reflectances * 10000) JPEG 2000 performs better and finally improves CCSDS.

\subsection{Classification Evaluation Results}

3.2.1. On-Board Compression. The "Les Landes-P03BS" and "Toulouse-Urban-T01BS" scenes were used to assess the impact of on-board compression on image classification. Only the CCSDS and JPEG 2000 standards were studied because the CNES compressors had very few scenarios with available results. To evaluate each classification its overall accuracy was first computed using a confusion matrix with independent ground-truth areas (as in [23]). The results of the two scenes (see Figures $7(\mathrm{a})$ and $7(\mathrm{~b})$ ) are quite different and the trends are not always in line with previous SNR results. This is interesting because it reminds us that it is necessary to use applied approaches, and not only noise or fidelity approaches, to evaluate the impact of onboard compression on the end-uses of the images. For the "Les Landes-P03BS" scene, the impact of JPEG 2000 compression on the overall accuracy is lower than that of CCSDS. Moreover, and at low compression ratios, equal distribution of the BR among bands gives similar or even better results than unequally distributed BR. These trends are contrary to those obtained with the SNR estimations. 


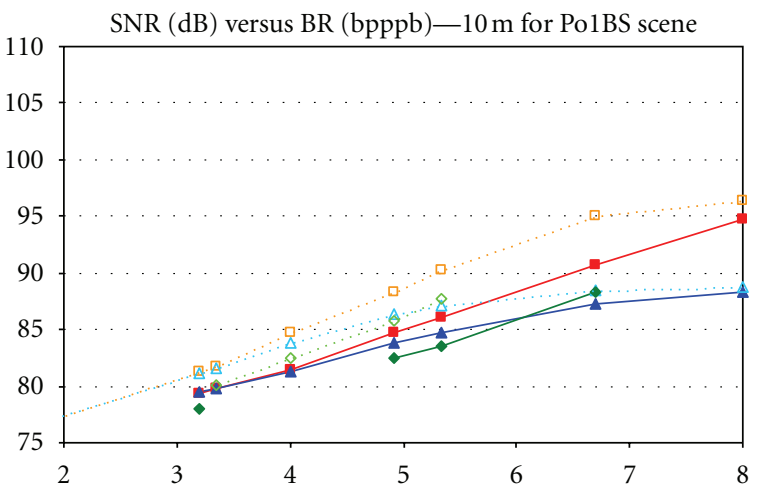

(a)

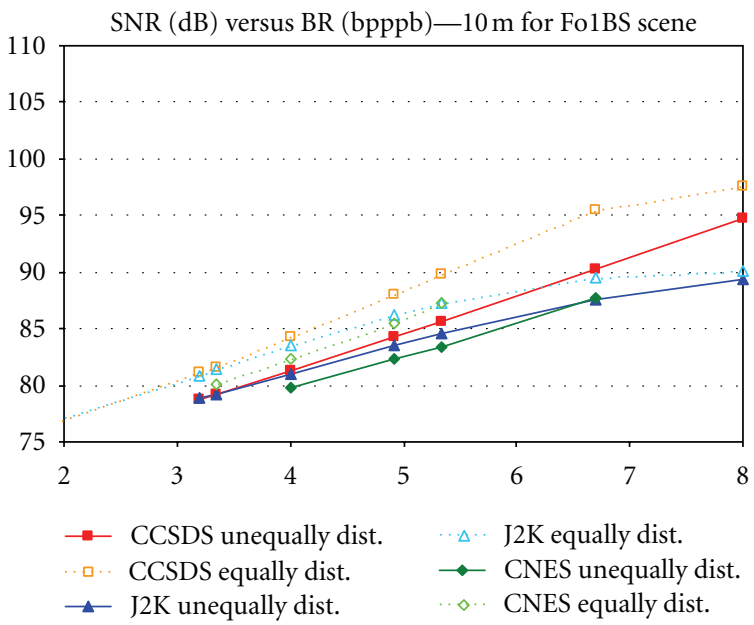

(c)

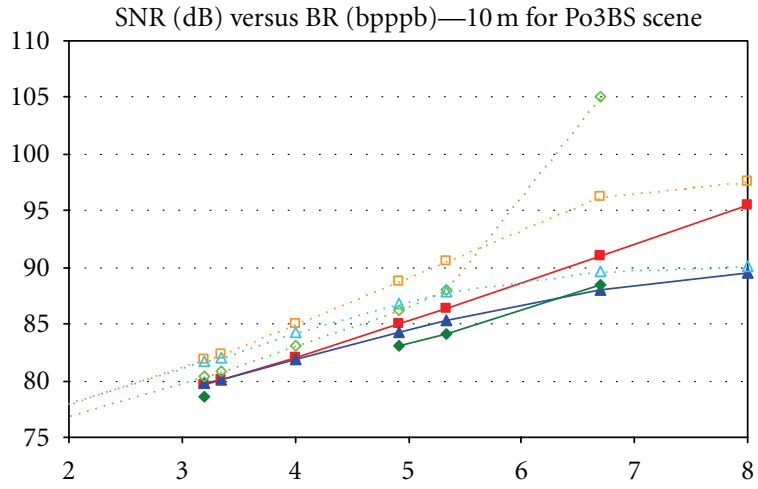

(b)

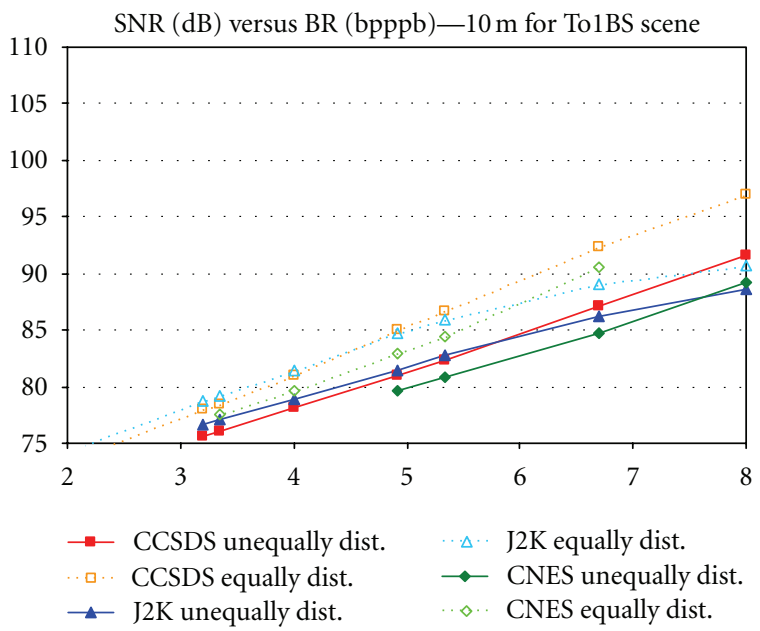

(d)

FIGURE 5: SNR (in dB) of $10 \mathrm{~m}$ bands in relation to the bit rate (in bpppb) of all the compression algorithms for all scenes at high bit rates. Two strategies are used to divide the BR among bands (at each compression ratio): unequal or equal distribution. (Please note that the $y$-axis is not the same as in Figures 4 and 6.)

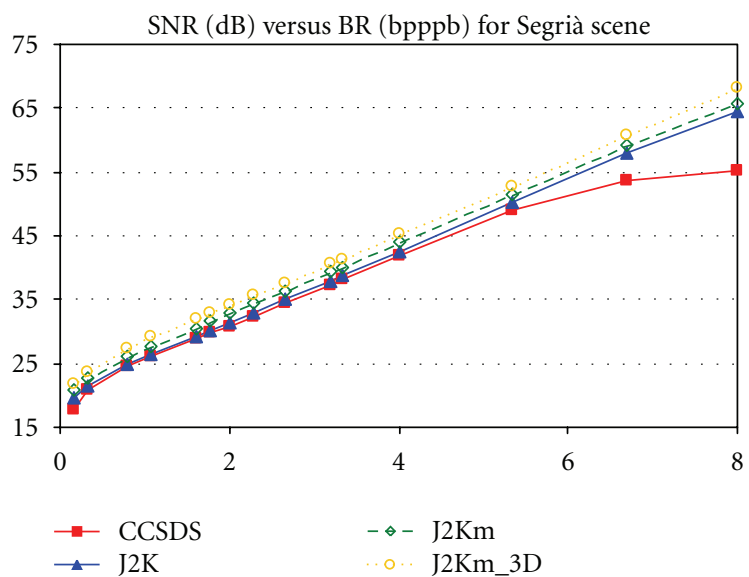

(a)

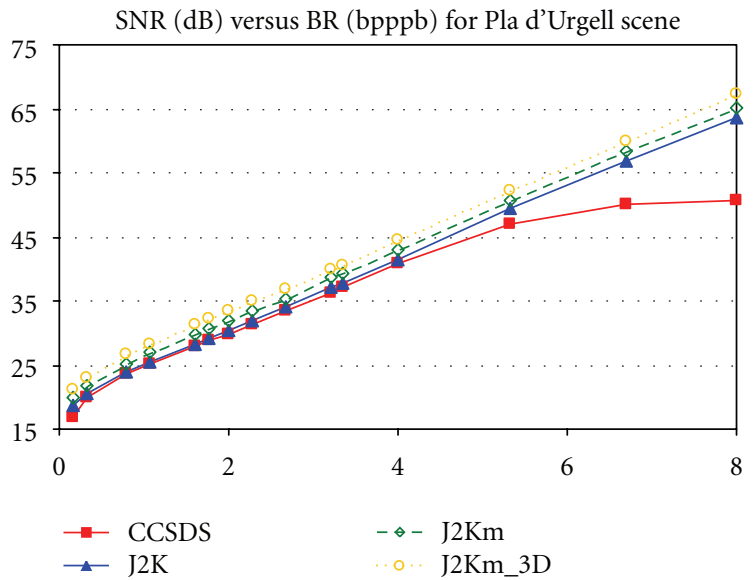

(b)

Figure 6: SNR (in $\mathrm{dB}$ ) in relation to the bit rate (in bpppb) obtained for the images recovered of the studied crop areas and for the four compression strategies. (Please note that the $y$-axis is not the same as in Figures 4 and 5.) 
For the "Toulouse-Urban-T01BS" scene, the trends show that CCSDS improves JPEG 2000 results and that unequally distributed BR is also better for the applied approach. The reason for the different behavior of the "Les Landes" scene may be the less fragmentation of the area, covered by extense fields, with a landscape that is robustly classified at several CRs or using different compression algorithms.

To examine this question in more depth and to obtain complementary evaluation information, the classification of noncompressed images was used as ground-truth for evaluating the compressed classifications (as in [24]). A confusion matrix can also be computed in this case to obtain the overall accuracy in relation to the original classification. The results are presented in Figures 7(c) and 7(d). Using this approach, the two areas show the same behavior as that observed before, that is, unequally BR distribution preserves more valuable information for the classification and obtain a higher overall accuracy. From this point of view, differences between the two compression standard are small but CCSDS performs slightly better. The differences between the two evaluation approaches outline the risk of not succeeding when the aim is to obtain a representative set of test polygons for the entire study area (as found in [25]).

3.2.2. User-Side Compression. The first approach shows the overall accuracy obtained using independent test areas (as in [23]). The results are shown in Figure 8. It can be seen that not much variation is produced in the overall accuracy in relation to the compression ratio. This is probably because the multitemporal approach that allows an optimal characterization of the categories even if some details on some bands are lost due to compression. Secondly, classifications were evaluated considering the classification of noncompressed images as ground truth (as in [24]). These results are shown in Figure 9. The trends in the overall accuracy computed with this second approach are clearer. In terms of the compression standards, CCSDS obtains the worst results followed by $\mathrm{J} 2 \mathrm{~K}, \mathrm{~J} 2 \mathrm{Km}$, and $\mathrm{J} 2 \mathrm{Km} \_3 \mathrm{D}$. However, some specific situations should be mentioned. For the IsoMM classifier at low compression ratios, CCSDS performs better than the other standard (in both studied areas). This is probably because JPEG 2000 produces a more smoothing effect (because more wavelet transform levels are used by default), and thus it loses the differences among neighbor pixels that are exploited by the unsupervised step of the classifier. This reminds us once again that the study of compression noise (by means of SNR or other metric computations) is not enough to assess the real impact on the applications of end users. Further research needs to be carried out especially regarding hybrid and unsupervised classifiers.

In terms of classification methods, the IsoMM classifier is most affected by compression. As an indicator we can determine the compression ratio at which the overall accuracy in relation to the original classification is lower than $90 \%$. For IsoMM this CR is around 3 or 4 for the Segrià area (i.e., 5.33 to $4 \mathrm{bpppb}$ ), and 4 or 4.79 for the Pla d'Urgell area (i.e., 4 to $3.34 \mathrm{bpppb}$ ), depending on the compression standards. However, for the MaxLike classifier it is possible to compress up to $15: 1$ in both areas (i.e., 1.07 bpppb) before this loss in the overall accuracy is reached (both areas).

Finally, some differences can also be concluded from the results when the two studied areas are compared. The Pla d'Urgell area is more affected by compression because it is more fragmented, as can be seen in the larger decrease in overall accuracy for all the compression algorithms and classification methods, especially at high compression ratios (e.g., at CR 20 : 1, i.e., 0.8 bpppb, using CCSDS standard, PU obtains 1.10 percentage points less than Segrià area).

\subsection{Discussion of the Results}

3.3.1. On-Board Compression. From the evaluation of the impact of on-board compression using the Sentinel-2 Image Performance Simulator and testing the CNES, CCSDS, and JPEG 2000 compressors it can be concluded that CCSDS obtains higher compression fidelity than JPEG 2000 at lower compression ratios (up to CR $5: 1$, i.e., $3.2 \mathrm{bpppb}$ ). This improvement is up to $8.01 \mathrm{~dB}$ in the best case ("Les LandesP03BS" scene compressed at CR 2: 1, i.e., 8 bpppb). However, at higher compression ratios (out of the scope of Earth Observation on-board missions), JPEG 2000 performs better than CCSDS.

It is also interesting to take into account that the larger the dynamic range, the smaller the difference between the two compression standards. Using an unequal distribution of BR among bands (e.g., CR 4.0, i.e., 4 bpppb, for $10 \mathrm{~m}$ bands and CR 2.2, i.e., $7.27 \mathrm{bpppb}$, for $20-60 \mathrm{~m}$ bands) improves the overall compression fidelity obtained (using any of the compression algorithms in this paper). The CNES compressor generally obtains lower compression fidelity than the two other compression standards. Moreover, as it is not a standardized algorithm, using it for Earth Observation on-board missions should be avoided.

In most cases the impact of compression on image classification follows the previous trends; however, we need to keep in mind that compression fidelity may not be enough to assess the impact of compression on end-user applications. Differences among compression algorithms and compression ratio distributions are smaller than differences in compression fidelity.

3.3.2. User-Side Compression. The evaluation of the impact of end-user compression using Landsat images and testing CCSDS and JPEG 2000 compressors showed that JPEG 2000 using 3D wavelet transform obtains higher compression fidelity than CCSDS and JPEG 2000 with different parameterization. This is due to the large dynamic range of the images (representing reflectances $* 10000$ ), which JPEG 2000 exploits better. This improvement is up to $16.63 \mathrm{~dB}$ in the best case (Pla d'Urgell area compressed at CR 2:1, i.e., 8 bpppb, with 3D JPEG 2000).

The overall accuracy obtained using independent test areas did not show much variation in relation to compression ratio in some scenes, probably because the low fragmentation of the area or the multitemporal approach that allows an optimal classification (even if some details are lost due to compression). 


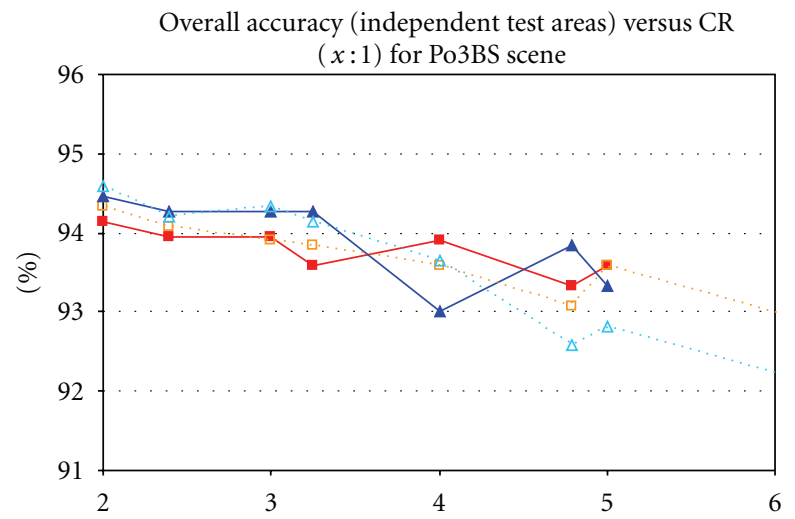

(a)

Overall accuracy (original classification) versus CR $(x: 1)$ for Po3BS scene

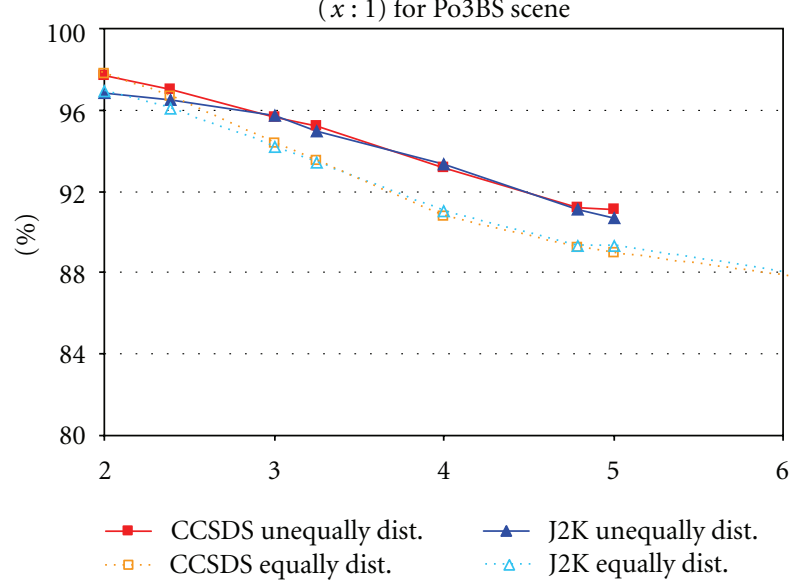

(c)

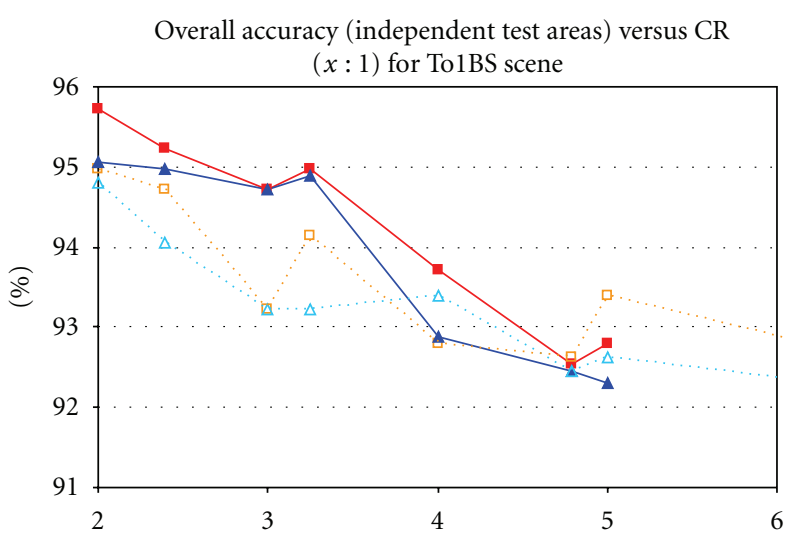

(b)

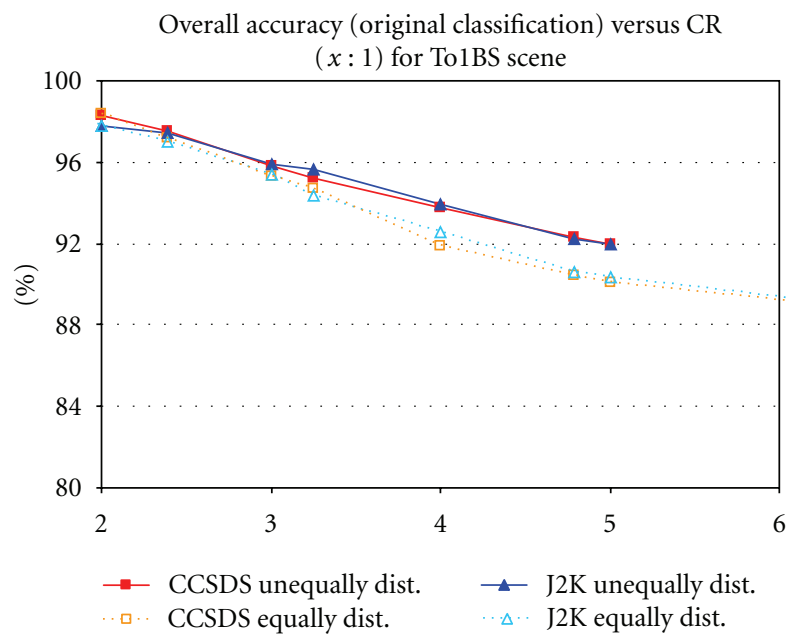

(d)

FIGURE 7: Overall accuracy (in \%) of classifications in relation to the compression ratio (CR, expressed as $x: 1$, e.g., $5: 1$ ) of the CCSDS and JPEG 2000 compression standards for the "Les Landes-P03BS" and “Toulouse-Urban-T01BS" scenes. As ground truth, the graphics (a and b) use independent test areas and the graphics (c and d) use the classification obtained without compression.

When the overall accuracy is calculated considering the classification of noncompressed images as ground truth, the trends are clearer. CCSDS obtains the worst results followed by $\mathrm{J} 2 \mathrm{~K}, \mathrm{~J} 2 \mathrm{Km}$, and $\mathrm{J} 2 \mathrm{Km} \_3 \mathrm{D}$. However, some particular situations should be mentioned. For the IsoMM classifier at low compression ratios CCSDS performs better than the other standard (in both studied areas). The study of the compression noise (by means of SNR or other metric computations) is not enough to assess the real impact of compression on end-user applications. Further research needs to be carried out especially regarding hybrid and unsupervised classifiers. The IsoMM classifier is the most affected by compression. The compression ratio at which the overall accuracy in relation to the original classification is lower than $90 \%$ is around CR 4.79 (i.e., 4 to $3.34 \mathrm{bpppb}$ ) in the best case for IsoMM, that is, $3.34 \mathrm{bpppb}$, at 15:1 for MaxLike, that is, $1.07 \mathrm{bpppb}$.

The more fragmented the area is, the higher the impact of compression is. For example, the Pla d'Urgell shows a larger decrease in overall accuracy for all the compression algorithms and classification methods, especially at high compression ratios (e.g., at CR $20: 1$, i.e., 8 bpppb, CCSDS standard, Pla d'Urgell obtains 1.10 percentage points less than the Segrià area).

\section{Conclusions}

The set of experiments performed to assess the impact of CCSDS and JPEG 2000 compression on Earth Observation scenarios shows that the relative performance of compression standards depends on the dynamic range of the image to be compressed as well as the image fragmentation.

For low dynamic ranges, CCSDS performs better than JPEG 2000 at most CRs; thus, if on-board compression is used, CCSDS is the best option, not only due to its better performance but also due to its smaller computational requirements. This improvement is up to $8.00 \mathrm{~dB}$ (Sen-2 simulated images using "Les Landes-P03BS" scene compressed at CR 2:1, i.e., 8 bpppb). When the dynamic range is larger or the compression ratio is higher (i.e., compressing images at the user side, representing reflectances * 10000, or compressing images on board at a high CR) 


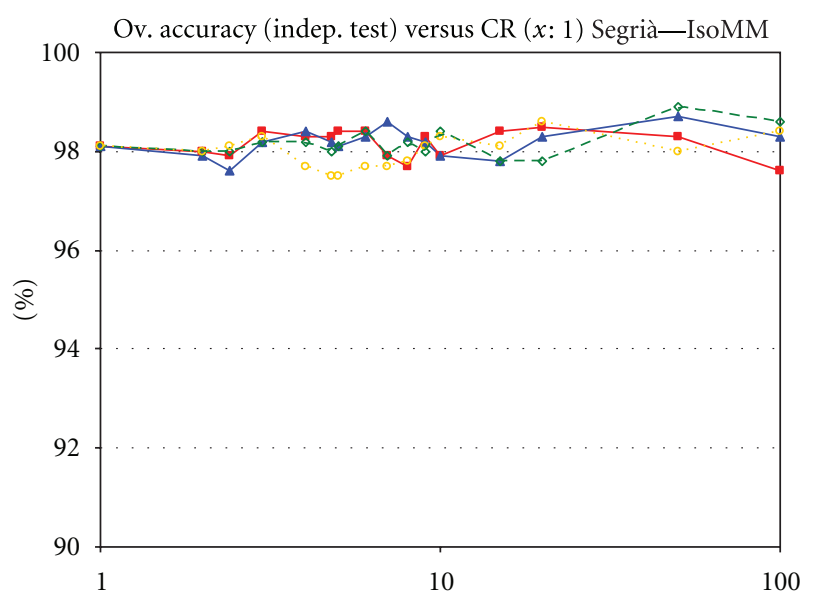

(a)

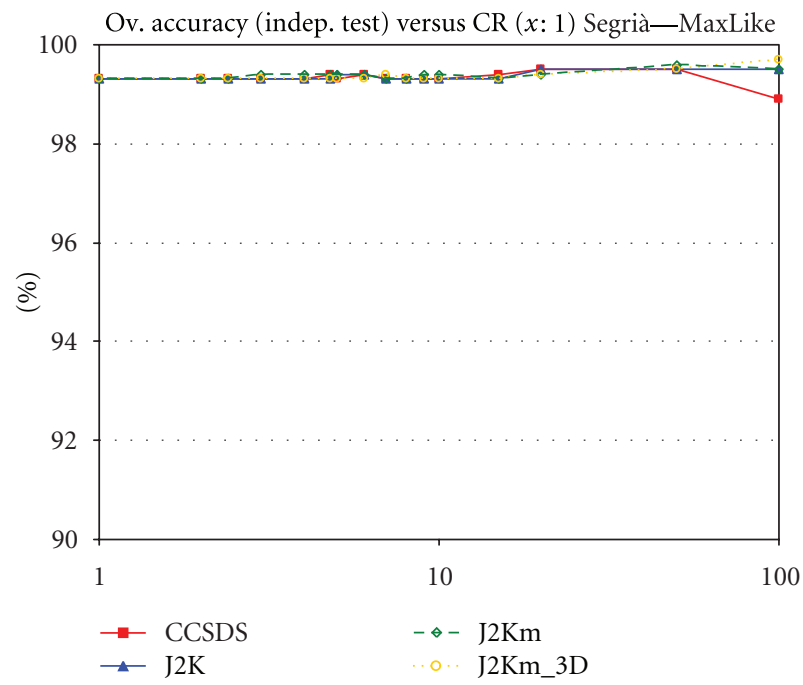

(c)

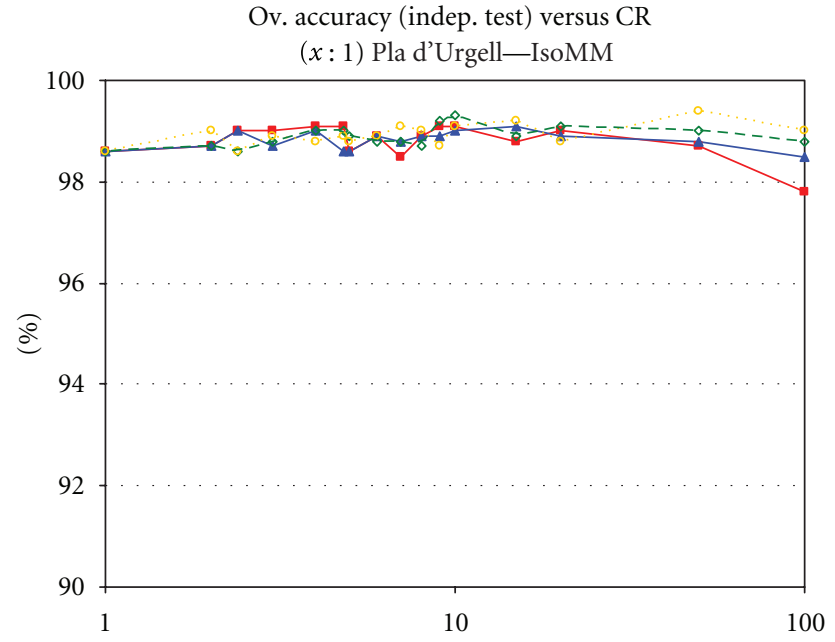

(b)

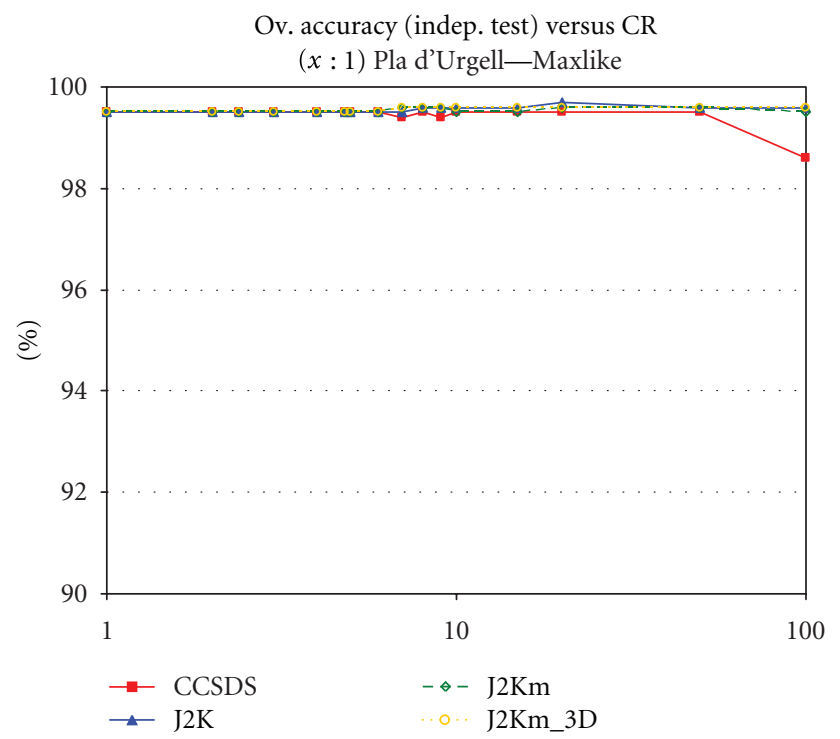

(d)

Figure 8: Overall accuracy (in \%) computed using independent test areas in relation to the compression ratio (CR, expressed as $x$ : 1 , e.g., $5: 1)$. The two study areas, two classifying methods, and four compression scenarios are shown.

JPEG 2000 performs better than CCSDS, especially if 3D DWT is applied. When compression is performed by end users themselves this compression approach should be used. The improvement is up to $16.63 \mathrm{~dB}$ in the best case (Pla d'Urgell area compressed at CR 2:1, i.e., 8 bpppb, with 3D JPEG 2000). Summarizing, CCSDS works better on board when images are not calibrated and the dynamic range is not large, while JPEG 2000 works better on ground with calibrated images with a larger dynamic range.

The impact of compression on classification usually follows compression noise trends, but differences among compression algorithms are smaller, particularly with IsoMM classifier. Further research needs to be carried out, especially regarding hybrid and unsupervised classifiers. IsoMM and maximum likelihood classifiers are more affected by compression. The compression ratio at which the overall accuracy in relation to the original classification is lower than $90 \%$ is around CR 4.79 for IsoMM (i.e., $3.34 \mathrm{bpppb}$ ) and at 15:1 for MaxLike (i.e., 1.07 bpppb). The more fragmented the area, the large the impact of compression. For example, the Pla d'Urgell shows a larger decrease in overall accuracy for all the compression algorithms and classification methods, especially at high compression ratios (e.g., at CR $20: 1$, i.e., 8 bpppb, using the CCSDS standard, Pla d'Urgell area obtains 1.10 percentage points less than the Segrià area).

To extend this research, a mathematical approach can be explored further to determine a rule that relates image characteristics to compression impact. This is quite an ambitious aim that can be approached theoretically by computing the compression noise of images with several dynamic ranges (e.g., modifying it manually) and with several image fragmentations (a measure of this image fragmentation or image information content should be further explored). Moreover, further research is needed on 


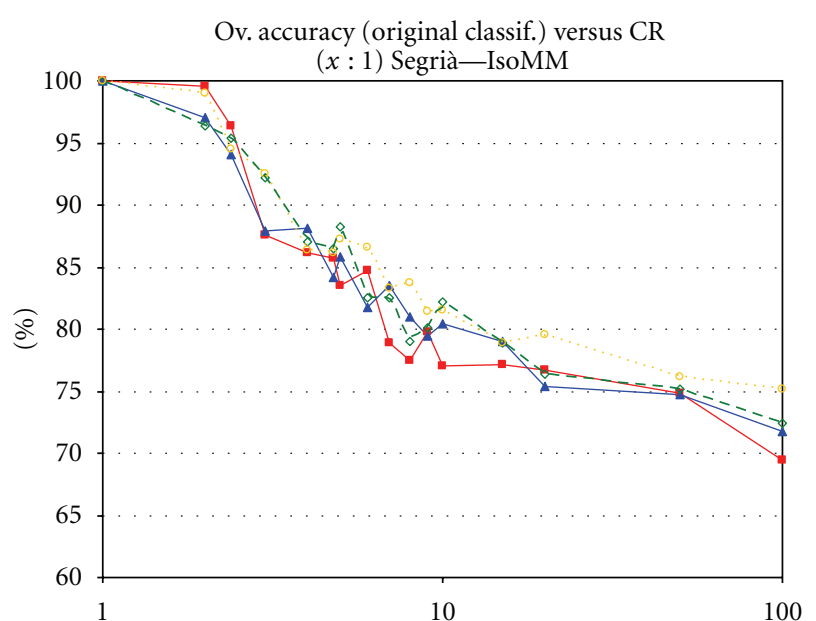

(a)

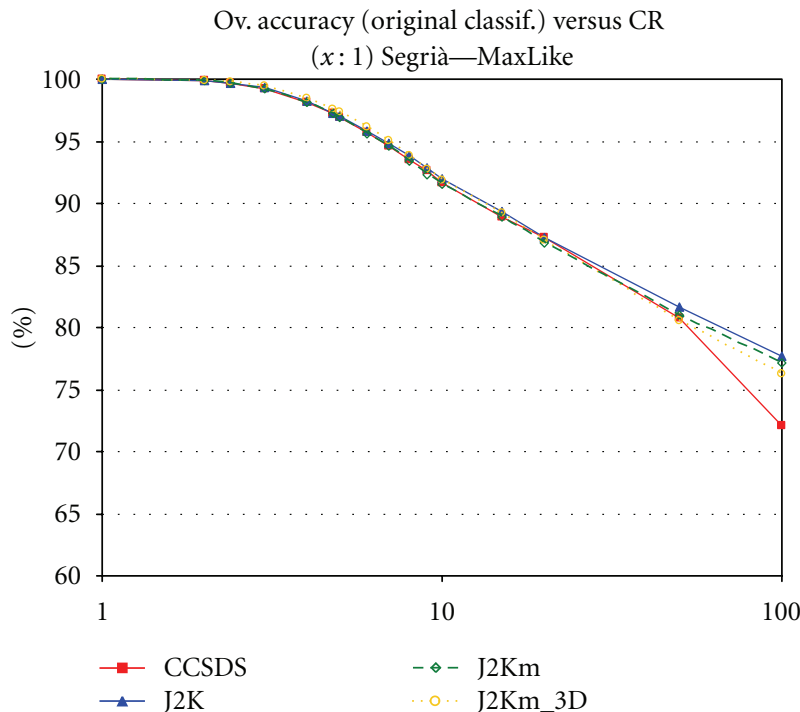

(c)

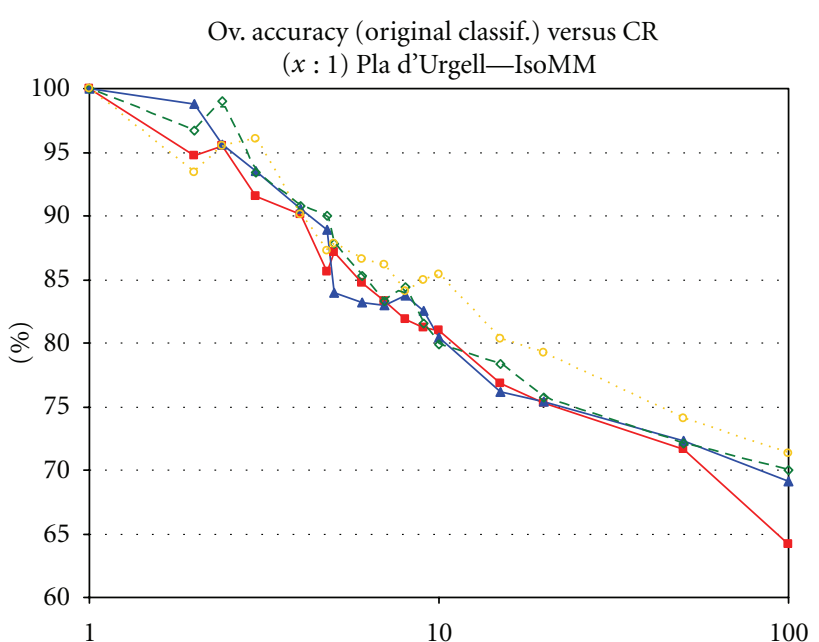

(b)

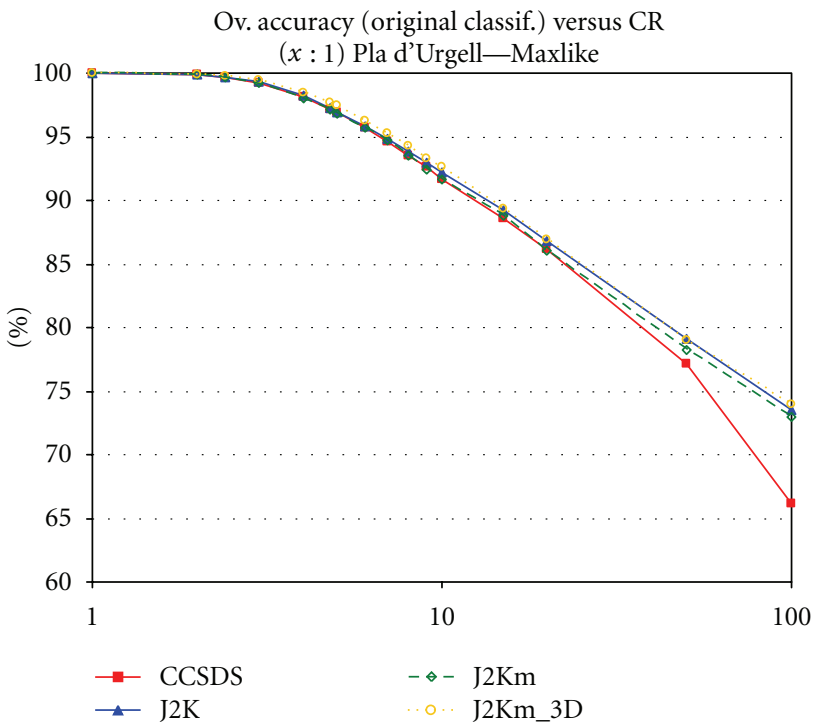

(d)

FIGURE 9: Overall accuracy (in \%) computed using classification of noncompressed images as ground-truth in relation to the compression ratio (CR, expressed as $x: 1$, e.g., $5: 1$ ). The two study areas, two classifying methods and four compression scenarios are shown.

the impact of compression when it is performed in ground segment processing.

\section{Acknowledgments}

The authors wish to thank the Sentinel-2 team at ESAESTEC and EADS Astrium who allowed us to use and extend the Sentinel-2 Image Performance Simulator. The authors would also like to thank their colleagues at the Department of Information and Communications Engineering (Autonomous University of Barcelona, UAB) who helped with compression, decompression, and metric computation tools. This work was supported in part by the Ministry of Education and Science and the FEDER funds through the Research Project TIN2009-14426-C02-02, as well as by the Commission for Universities and Research (CUR) at the Department of Innovation, Universities and
Enterprise (DIUE) of the Catalan Government (SGR20091511 and BE2009-2-00426). X. Pons is recipient of an ICREA Acadèmia Excellence in research grant (2011-2015).

\section{References}

[1] G. Yua, T. Vladimirova, and M. N. Sweeting, "Image compression systems on board satellites," Acta Astronautica, vol. 64, no. 9-10, pp. 988-1005, 2009.

[2] D. J. Maguire and P. A. Longley, "The emergence of geoportals and their role in spatial data infrastructures," Computers, Environment and Urban Systems, vol. 29, no. 1, pp. 3-14, 2005.

[3] J. Masó, K. Pomakis, and N. Julià, OpenGIS ${ }^{\circledR}$ Web Map Tile Service Implementation Standard, v. 1.0.0, Open Geospatial Consortium Inc. Document reference number 07-057r7, 2010, http://www.opengeospatial.org/standards/wmts. 
[4] ISO/IEC 15444-1:2004, "Information technology-JPEG 2000 image coding system: core coding system," Geneva, Switzerland, 2004.

[5] CCSDS, Image Data Compression, recommendation for space data system standards, CCSDS 122.0-B-1, 2005, http://public.ccsds.org/publications/archive/122x0b1c2.pdf.

[6] Sentinel-2 team, GMES Sentinel-2 Mission Requirements Document, EOP-SM/1163/MR-dr, Issue 2 Rev. 1, March 2010, http://esamultimedia.esa.int/docs/GMES/Sentinel-2_MRD. pdf.

[7] Sentinel-2 team, GMES Sentinel-2 Image Performance Simulator Description, S2-TN-ASG-SY-2002, Issue 1 Rev. 1, ESA confidential, February 2007.

[8] CEFLES team, CEFLES-2 Final Report, contract no. 20801/07/I-LG and 20802/07/I-LG, June 2009, http://earth.esa .int/campaigns/index.htm.

[9] M. Cabral, "WhiteDwarf compression tester user's manual," European Space Agency, TEC-EDP, 2009.

[10] H. Wang, An implementation of CCSDS 122.0-B-1 Recommended Standard, March 2008, http://hyperspectral.unl.edu/.

[11] GICI group, "BOI user manual," Department Group on Interactive Coding of Images, Autonomous University of Barcelona, September 2005, http://www.gici.uab.cat/BOI/.

[12] X Pons, MiraMon: Geographical Information System and Remote Sensing Software, Version 6, 2008, http://www.creaf .uab.cat/miramon/.

[13] ITTVIS, “ENVI software version 4.3,” 2006, http://www.ittvis .com/ProductServices/ENVI.aspx.

[14] H. Xie, Y. Q. Tian, J. A. Granillo, and G. R. Keller, "Suitable remote sensing method and data for mapping and measuring active crop fields," International Journal of Remote Sensing, vol. 28, no. 2, pp. 395-411, 2007.

[15] G. Chirici, P. Corona, M. Marchetti, and D. Travaglini, "Testing Ikonos and Landsat 7 ETM+ potential for standlevel forest type mapping by soft supervised approaches," in Advances in Forest Inventory for Sustainable Forest Management and Biodiversity Monitoring, P. Corona, M. Köhl, and M. Marchetti, Eds., Forestry Sciences, pp. 71-86, Kluwer Academic Publishers, Dordrecht, The Netherlands, 2003.

[16] V. Palà and X. Pons, "Incorporation of relief into geometric corrections based on polynomials," Photogrammetric Engineering \& Remote Sensing, vol. 61, no. 7, pp. 935-944, 1995.

[17] X. Pons and L. Solé-Sugrañes, "A simple radiometric correction model to improve automatic mapping of vegetation from multispectral satellite data," Remote Sensing of Environment, vol. 48, no. 2, pp. 191-204, 1994.

[18] D. Taubman, Kakadu Survey Documentation, last updated for Version 5.0, UNSW, October 2005, http://www.kakadusoftware.com.

[19] A. Zabala, J. Gonzalez-Conejero, J. Serra-Sagrista, and X. Pons, "JPEG2000 encoding of images with NODATA regions for remote sensing applications," Journal of Applied Remote Sensing, vol. 4, no. 1, Article ID 041793, 2010.

[20] P. Serra, X. Pons, and D. Saurí, "Post-classification change detection with data from different sensors: some accuracy considerations," International Journal of Remote Sensing, vol. 24, no. 16, pp. 3311-3340, 2003.

[21] P. Serra, G. Moré, and X. Pons, "Cadastre land-cover enrichment using remote sensing data: thematic accuracy consequences of being more or less conservative from a pixel and from a polygon perspective," Photogrammetric Engineering \& Remote Sensing, vol. 75, no. 12, pp. 1441-1449, 2009.
[22] G. M. Foody, "Status of land cover classification accuracy assessment," Remote Sensing of Environment, vol. 80, no. 1, pp. 185-201, 2002.

[23] S. E. Qian, A. Hollinger, M. Bergeron et al., "A multidisciplinary user acceptability study of hyperspectral data compressed using an on-board near lossless vector quantization algorithm," International Journal of Remote Sensing, vol. 26, no. 10, pp. 2163-2195, 2005.

[24] E. Choi, S. Lee, and C. Lee, "Effects of compression on the classification of hyperspectral images," in Mathematics and Computers in Science and Engineering, N. E. Mastorakis, V. Mladenov, Z. Bojkovic et al., Eds., pp. 541-546, Heraklion, Greece, 2008.

[25] A. Zabala, C. Cea, and X. Pons, "Segmentation and thematic classification of color orthophotos over non-compressed and JPEG 2000 compressed images," International Journal of Applied Earth Observation and Geoinformation, vol. 15, pp. 92104, 2012. 

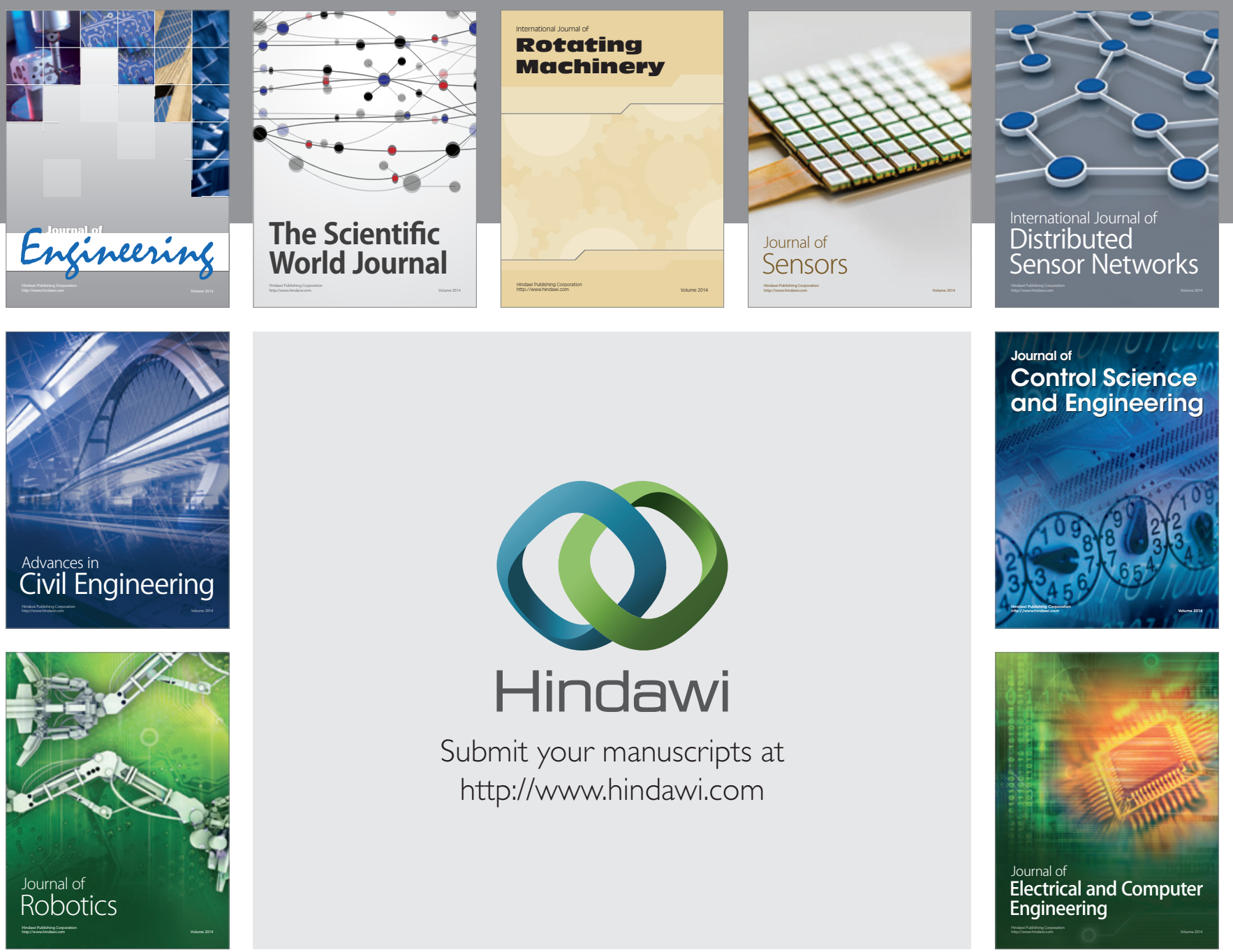

Submit your manuscripts at

http://www.hindawi.com
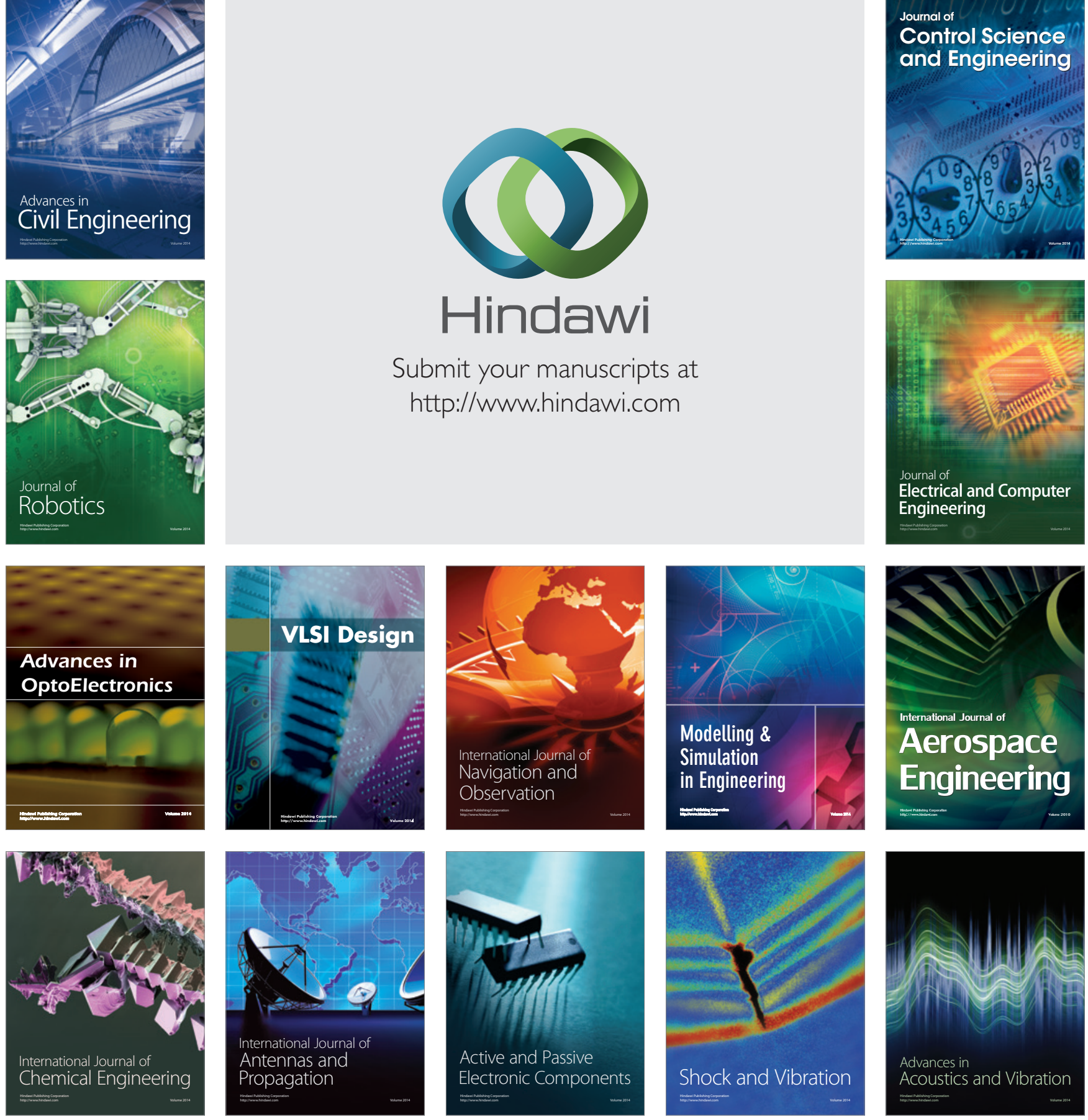